\title{
Serological Monitoring on Layer Farms with Specific Pathogen-Free Chickens
}

\author{
Kozo TAKASE ${ }^{1)}$, Yasushi MURAKAWA ${ }^{2)}$, Rikako ARIYOSHI ${ }^{2}$, Sin-ichi ERIGUCHI ${ }^{2)}$, Takaaki SUGIMURA ${ }^{1)}$ and \\ Hideo FUJIKAWA ${ }^{2}$ \\ ${ }^{1)}$ Department of Veterinary Microbiology, Faculty of Agriculture, Kagoshima University, 1-21-24 Korimoto, Kagoshima 890-0065 and \\ ${ }^{2)}$ The Chemo-Sero-Therapeutic Research Institute, 1-6-1 Okubo, Kumamoto 860-8568, Japan
}

(Received 26 June 2000/Accepted 23 August 2000)

ABSTRACT. To monitor the existence of avian pathogens in laying chicken flocks, specific pathogen-free (SPF) chickens were introduced into two layer farms and reared with laying hens for 12 months. SPF chickens were bled several times after their introduction and examined for their sero-conversion to avian pathogens. As a result, antibodies to eight or ten kinds of pathogens were detected in SPF chickens on each farm. Antibodies to infectious bronchitis virus (IBV), avian nephritis virus, Mycoplasma gallisepticum and M. synoviae were detected early within the first month. Antibody titer to IBV suggested that the laying chickens were infected with IBV repeatedly during the experiment on both farms. However, antibodies to infectious bursal disease virus and 6 pathogens were not detected. KEY WORDS: avian pathogen, layer farm, serological monitoring.

J. Vet. Med. Sci. 62(12): 1327-1329, 2000

Many pathogens such as avian encephalomyelitis virus (AEV), infectious bronchitis virus (IBV), Hemophiles paragarinarum (Hpg), Mycoplasma gallisepticum (MG) etc. are known to be causal agents of a decline in egg production and egg quality of laying hens. On almost all farms, vaccination during the laying period is not preferable because it induces stresses in many hens and might then cause a drop in egg production. In Japan, recently, many oil adjuvanted killed vaccines have been used for pullets before they begin to lay eggs to give hens a high and long-persistent immunity [1]. However, we have occasionally observed some egg production problems in these chicken flocks even though they were fully vaccinated before laying age. In general, antibodies are examined to guess or confirm the possibility of infection with pathogens which cause egg production problems. But, it is not easy to understand the serological data of laying hens, because many antibodies produced by vaccinations or natural infections at a young age, can usually last and be detected in many of them.

Therefore, we try to use SPF chickens for monitoring the actual existence of pathogens among laying hens. In this study, SPF chickens were introduced as monitors into two layer farms where they were in contact with laying hens for 12 months. They were bled at several intervals and examined serologically.

Two layer farms (Farms A and B) in Kumamoto prefecture were employed. Farm A consisted of 22 open coops and 4 windless coops in which a total about 140,000 hens of older than 110 days of age had been reared. Water was supplied by a nipple-drinker system. The test was conducted in one open coop which contained about 4,000 laying hens. They had been vaccinated against Marek's disease virus (MDV), Newcastle disease virus (NDV), fowl pox virus (FPV), IBV, infectious bursal disease virus (IBDV), Hpg (types A and C), and MG until 65 days of age, then introduced into farm A at 100 days of age and reared until 560 days of age. At 137 days of age, ten SPF chickens of 120 days of age were carried into this open coop and reared with laying hens for 12 months. The SPF chickens were divided and placed into 5 cages (two birds per cage) among laying hens.

Farm B had 3 windless coops which contained a total of about 100,000 hens aged from 120 to 750 days. They had been vaccinated against MDV, NDV, FPV, IBV, IBDV, Hpg (types $\mathrm{A}$ and $\mathrm{C}$ ), and MG until 60 days of age, then introduced into farm B at 120 days of age. Tests were begun in one windless coop where 170-day-old laying hens were reared. Ten SPF chickens of 145 days of age were placed into two cages (five birds per cage) near the entrance of the house and reared with laying hens for 12 months.

SPF White-Leghorn chickens were obtained from an SPF chicken breeding flock which was maintained at the authors' laboratories and confirmed to be free from many avian pathogens including the 12 viruses and four bacteria listed in Table 1.

The SPF chickens introduced into the two layer farms were bled $0,1,2,4,8$, and 12 months after introduction, for serological tests. Ten laying hens at each farm were also bled at the beginning of the experiments. Neither SPF nor laying hens of both farms had any vaccination during the experiments. The antibodies to AEV, NDV, MDV, FPV, IBV, IBDV, ANV, infectious laryngotracheitis virus (ILTV), fowl adenovirus (FAV), egg drop syndrome-1976 virus (EDS76V), chicken anemia virus (CAV), avian reovirus (ARV), turkey rhinotracheitis virus (TRTV), MG, M. synoviae (MS), and Hpg $(\mathrm{A}, \mathrm{C})$ were examined by the ordinary methods of agar gel precipitation, agglutination, hemagglutination inhibition, florescent antibody or neutralization tests, as described before [2] using antigen-strains shown in Table 1.

The serological results are shown in Table 2. At the start, on farms A and B, laying hens were antibody-positive to 12 and 13 pathogens, respectively. In SPF chickens on farm A, antibodies to IBV, MDV, CAV, ANV, AEV, TRTV, FAV, MG, MS, and Hpg (A) were detected. On the other hand, on farm B, IBV, MDV, CAV, ANV, FAV, Hpg (A), MG, and 
Table 1. Materials and methods for serology test

\begin{tabular}{lcc}
\hline $\begin{array}{l}\text { Virus or bacteria (strain) } \\
\text { used for antigen }\end{array}$ & $\begin{array}{l}\text { Test } \\
\text { method }^{a}\end{array}$ & Level for positive titer \\
\hline ILTV (NS-175) & SN & $\begin{array}{l}\geq 0.75 \text { (Neutralizing index) } \\
\geq 1: 2\end{array}$ \\
IBV (Nerima) & HI & $\geq 1: 5$ \\
NDV (B1), EDS-76V *KE80), & IFA & $\geq 1: 20$ \\
Hpg-A (221), Hpg-C (S1) & \\
ANV (G-4260), CAV (Gifu-1), & \\
TRTV (CVL14/1), FPV (Nishigahara) & IFA & \\
IBDV (J1), ARV (Uchida), AEV (VR) & AGP & Appearance of precipitin-line \\
FAV (Ote), MDV (Alabama) & AGG & $\geq 1: 1$ \\
MG (S6), MS (WVU1853) &
\end{tabular}

a) SN: Serum neutralization test, HI: Hemagglutination test, IFA: Indirect immunofluorescence assay, AGP: Agar gel precipitation test, AGG: Agglutination test.

Table 2. Sero-conversion of SPF chickens introduced into Farms A and B

\begin{tabular}{|c|c|c|c|c|c|c|c|c|}
\hline \multirow{2}{*}{ Farm } & \multirow{2}{*}{ Antigen } & \multirow{2}{*}{$\begin{array}{l}\text { Laying } \\
\text { hens }^{\text {a) }}\end{array}$} & \multicolumn{6}{|c|}{ Months after introduction of SPF chickens } \\
\hline & & & 0 & 1 & 2 & 4 & 8 & 12 \\
\hline \multicolumn{9}{|c|}{ (Number of chickens examined) } \\
\hline \multirow{11}{*}{ A } & & 10 & 10 & 10 & 10 & 10 & 9 & 6 \\
\hline & IBV & $10^{\mathrm{b})}$ & $-c^{c)}$ & 1 & 7 & 8 & 5 & 6 \\
\hline & MDV & 6 & - & - & - & - & 1 & 3 \\
\hline & CAV & 10 & - & 2 & - & - & 1 & 2 \\
\hline & ANV & 7 & - & 4 & 3 & 8 & 4 & 2 \\
\hline & AEV & - & - & - & - & 9 & 8 & 5 \\
\hline & FAV & 10 & - & - & - & 1 & - & - \\
\hline & TRTV & - & - & - & - & - & 9 & 6 \\
\hline & MG & 8 & - & 2 & 5 & 6 & 9 & 6 \\
\hline & MS & 6 & - & 1 & 3 & 6 & 7 & 3 \\
\hline & $\operatorname{Hpg}(\mathrm{A})$ & 9 & - & 1 & 2 & 1 & - & - \\
\hline \multicolumn{9}{|c|}{ (Number of chickens examined) } \\
\hline \multirow{9}{*}{ B } & & 10 & 10 & 10 & 10 & 10 & 9 & 8 \\
\hline & IBV & 10 & - & 7 & 10 & 10 & 8 & NT \\
\hline & MDV & 5 & - & - & - & - & - & 2 \\
\hline & CAV & 7 & - & 2 & - & - & 2 & - \\
\hline & ANV & 3 & - & 1 & 4 & 3 & 2 & 3 \\
\hline & FAV & - & - & - & - & - & 1 & 2 \\
\hline & MG & 7 & - & 6 & 9 & 7 & 9 & 8 \\
\hline & MS & 5 & - & 6 & 6 & 4 & 7 & 7 \\
\hline & $\operatorname{Hpg}(\mathrm{A})$ & 6 & - & - & - & 1 & 1 & 1 \\
\hline
\end{tabular}

a) Antibodies of laying hens were examined at the time the study was commenced.

b) Antibody-positive number.

c) All negative.

MS were positive. Especially, on both farms, sero-conversion of SPF chickens to IBV, ANV, MG, and MS were detected early within 1 or 2 months after introduction. AEV and TRTV were positive on farm A, but not on farm B. FAV and Hpg (A) were at very low positivity rates on both farms. On both farms, antibodies to NDV, IBDV, FPV, EDS-76V, $\mathrm{ARV}$, and $\mathrm{Hpg}(\mathrm{C})$ were not developed throughout the experiment. Neutralizing antibody titer to the Nerima strain of IBV, Mass. type, was examined and the results are shown in Table 3. On both farms, antibodies to the Nerima strain were detected within the first month and lasted until the end of the experiment, although some of the titers went up or down during the test period.

During the 12 month-experiment, six SPF chickens died. The causes of their deaths were not examined here. On the last day of this study, the surviving SPF chickens were necropsied. But, we could not find any pathological lesions in the SPF chickens. Egg production of SPF chickens was not recorded on both farms. However, according to the farmrecord, any clinical symptoms or egg drop problems were not 
Table 3. Neutralizing antibody titer to the Nerima strain of IBV in sera of SPF chickens introduced into Farms A and B

\begin{tabular}{cccccccc}
\hline \multirow{2}{*}{ Farm } & Bird & \multicolumn{6}{c}{ Months after introduction of SPF chickens } \\
\cline { 2 - 7 } & No. & 0 & 1 & 2 & 4 & 8 & 12 \\
\hline \multirow{6}{*}{ A } & 569 & $<2^{\text {a) }}$ & $<2$ & 32 & 16 & 4 & 8 \\
& 570 & $<2$ & $<2$ & 128 & 64 & NT & NT \\
& 572 & $<2$ & $<2$ & 4 & 16 & 4 & 4 \\
& 573 & $<2$ & $<2$ & 64 & 32 & 8 & 64 \\
& 574 & $<2$ & $<2$ & 16 & 4 & $<2$ & NT \\
& 575 & $<2$ & $<2$ & $<2$ & 16 & 64 & 16 \\
& 576 & $<2$ & 16 & 32 & 16 & $<2$ & 16 \\
& 577 & $<2$ & $<2$ & 16 & 8 & $<2$ & 32 \\
& 579 & $<2$ & $<2$ & $<2$ & $<2$ & $<2$ & NT \\
& 580 & $<2$ & $<2$ & 4 & $<2$ & 8 & NT \\
\hline \multirow{6}{*}{} & 591 & $<2$ & 32 & 32 & 8 & 256 & NT \\
& 592 & $<2$ & 2 & 64 & 16 & 16 & NT \\
& 593 & $<2$ & 4 & 8 & 64 & 256 & NT \\
& 594 & $<2$ & 8 & 256 & 64 & 256 & NT \\
& 595 & $<2$ & $<2$ & 8 & 8 & 16 & NT \\
B & 596 & $<2$ & 16 & 2 & 16 & 256 & NT \\
& 597 & $<2$ & $<2$ & 16 & 64 & $<2$ & NT \\
& 598 & $<2$ & 4 & 128 & NT & NT & NT \\
& 599 & $<2$ & 4 & 64 & 128 & 128 & NT \\
& 600 & $<2$ & $<2$ & 2 & 16 & 4 & NT \\
\hline
\end{tabular}

a) Neutralizing antibody titer (1:n).

observed in both laying flocks during the experiments. The influence of the pathogens detected by sero-conversion of SPF chickens might be small on egg production of each laying flock.

We conducted a survey on a non-vaccinated broiler farm and reported previously that many pathogens infected the broiler chickens [2]. In this study, it was also revealed that the laying chicken farms were contaminated with many pathogens during the laying period, too.

Antibodies to IBV, MG, and MS were detected with highly positive rates soon after the experiments started. It is commonly known that MG and MS persist for a long period in contaminated chicken flocks. In the two flocks from this study, MG and MS infected the SPF chickens soon after they were introduced. This shows that the occurrence of excretion of MG and MS from laying hens was common in both flocks.

The laying hens in both flocks were vaccinated fully against IBV; nevertheless, infection by IBV seemed to be repeated. The sero-types of the IBVs which invaded the flocks are not known, although suggesting that the viruses share same antigen with the Nerima strain used in the neutralization test. To discuss the sero-types of IBVs more in detail, the isolation of IBV will be necessary.

ANV has been isolated from young chickens [3]; however, ANV isolation from laying chickens has not yet been reported. In our experiments, the SPF chickens showed seroconversion soon after contact with laying hens. This suggests that the infection and excretion of ANV in laying chicken flocks may occur naturally.

CAV and IBDV are dominant viruses in the field. They infect young chickens in early days of age immediately after the maternal antibody has declined and easily transmit horizontally in young chicken flocks. In the present study, the existence of CAV was confirmed in both flocks, while that of IBDV was not observed throughout the experiments. Both farms seem to be free from IBDV. The laying hens of both flocks already had antibodies to IBDV when they were carried into the farms. Therefore, IBDV might neither be excreted from them nor infect them. Almost all laying chicken farms may be free from IBDV even though they are positive serologically.

The monitoring of chicken farms using SPF chickens will be a good tool to examine the actual contamination of avian pathogens in farms. In particular, it will be very useful in the case where chickens of the farms already had antibodies to the targets by vaccination or natural infection. However, we should be careful when the SPF chickens are introduced into commercial chicken farms, because they will play a role in amplifying the pathogens which exist or remain in the farms.

ACKNOWLEDGEMENTS. The authors wish to thank Dr. Y. Uchinuno of Tsuboi Breeding Farm Co. Ltd. and Dr. T. Mihara of Mihara Poultry Farm for their kind support of this study.

\section{REFERENCES}

1. Honda, T. 1998. Sci. Rep. Chemo-Sero-Therap. Res. Inst. 7: 117.

2. Murakawa, Y., Takase, K., Murakami, T., Maeda, H. and Uchinuno, Y. 1998. J. Jpn. Soc. Poult. Dis. 34: 43-48.

3. Takase, K. and Yamamoto, M. 1992. J. Jpn. Vet. Med. Assoc. 45: 639-642. 